\title{
THE UNDECIDABILITY OF A FUNDAMENTAL PROBLEM IN CLUSTER SET THEORY
}

\author{
J. A. EIDSWICK
}

\begin{abstract}
The undecidability of the existence of a nonmetrizable normal picket fence space is established and used to establish the undecidability of the following statement: Any family of approach curves (approaching a point in $\mathbf{R}^{2}$ ) along which cluster sets can be arbitrarily preassigned has a nonintersecting truncation.
\end{abstract}

1. Let $\Gamma$ denote the set of all curves in $\mathbf{R}^{2}$ that approach the origin, and for $\gamma \in \Gamma$ and $f: \mathbf{R}^{2} \rightarrow \mathbf{R}$, let $C(f, \gamma)$ denote the cluster set of $f$ at 0 along the curve $\gamma$ (i.e., $C(f, \gamma)=\left\{\lambda\right.$ : there exists a sequence $\left\{P_{n}\right\}$ of distinct points on $\gamma$ such that $P_{n} \rightarrow 0$ and $\left.\left.f\left(P_{n}\right) \rightarrow \lambda\right\}\right)$. A search in [2] for large subsets $\Gamma_{0}$ of $\Gamma$ along which cluster sets could be arbitrarily preassigned led to the following conjecture:

(T) A subfamily $\Gamma_{0}$ of $\Gamma$ has the property that for any given collection $\left\{C(\gamma): \gamma \in \Gamma_{0}\right\}$ of closed sets there exists a function $f$ such that $C(f, \gamma)=C(\gamma)$ for every $\gamma$ in $\Gamma_{0}$ if and only if $\Gamma_{0}$ has a nonintersecting truncation.

In [3] statement $(\mathrm{T})$ was shown to be equivalent to the restriction of the normal Moore space conjecture to a special class of spaces. Consequently (see [3]), (T) is consistent with the usual axioms of set theory (ZFC). The purpose of this paper is to show that the negation of $(T)$ is also consistent. This will be done by establishing the existence of a nonmetrizable normal picket fence space. Such a space will then be transformed into a counterexample to (T). Martin's Axiom plus the negation of the continuum hypothesis (MA $+\neg \mathrm{CH}$ ) will be assumed (cf. [8]).

2. A nonmetrizable normal picket fence space. As in [3], for $S \subset \mathbf{R}$ and $m: S \rightarrow \mathbf{R}$, let $(S, m)$ denote the family of half-lines $y=m(s) x+s, x \geqslant 0, s$ $\in S$, and let $X(S, m)$ denote the associated picket fence space (the points of $X(S, m)$ are the points of the right half-plane $x>0$ together with the points of $S$; the right half-plane has the discrete topology and basic neighborhoods of points $s$ of $S$ are the truncated lines $y=m(s) x+s, 0 \leqslant x<\tau, \tau>0)$.

The main difference between these spaces and the familiar tangent disk

Presented to the Society, January 22, 1976 under the title $A$ nonmetrizable normed picket fence space; received by the editors February 9, 1976 and , in revised form, April 1, 1976.

AMS (MOS) subject classifications (1970). Primary 26A15, 54D15, 54E30, 54E35; Secondary 02K05, 04A30, 26A30.

Key words and phrases. Cluster sets, approach curves, Moore spaces, picket fence spaces, metrizability, infinite derivatives. 
spaces (see [9]) is that these spaces generally do not have the countable cellularity property on the discrete subspace $S$ (for an extreme case, see [4]). Hence, the usual method of obtaining a normal noncollectionwise Hausdorff space from a $Q$-set does not apply to picket fence spaces. Also, it is not at all clear how a nonmetrizable normal space formed from triangular disks or wedges could be converted into one formed from pickets. Thus, it appears that a special approach like the one given below is necessary.

\section{THEOREM (MA $+\neg \mathrm{CH})$. There exists a nonmetrizable normal picket fence} space.

Proof. Let $S$ be any set of real numbers of power $\boldsymbol{\aleph}_{1}$. By [7, pp. 38-40] and [5, p. 154], MA $+\neg \mathrm{CH}$ implies that $S$ is a $Q$-set and has Lebesgue measure zero. Note that $X(S, m)$ is normal no matter how $m$ is defined (cf. [1, Example E]).

Let $\phi$ be a function from $\mathbf{R}$ to $\mathbf{R}$ whose derivative is equal to $-\infty$ at every point of $S$. This is possible because $S$ has measure zero (see [6, p. 214]). Let $m$ be the restriction of $\phi$ to $S$. Observe that if $X(S, m)$ were collectionwise Hausdorff, then for some uncountable subset $A$ of $S$ and some positive number $\varepsilon$, the family $(A, m)$ would be nonintersecting on the strip $0<x<\varepsilon$. The following argument shows that this is not the case.

Let $A$ be an uncountable subset of $S$ and let $a$ be a point of $A$ which is also a limit point of $A$. Since $\phi^{\prime}(a)=-\infty$, there exists a sequence $\left\{c_{n}\right\}$ in $A$ such that $\left(\phi\left(c_{n}\right)-\phi(a)\right) /\left(c_{n}-a\right) \leqslant-n$ for every $n$. Hence, there exist sequences $\left\{a_{n}\right\}$ and $\left\{b_{n}\right\}$ in $A$ such that $0<\left(b_{n}-a_{n}\right) /\left(m\left(a_{n}\right)-m\left(b_{n}\right)\right)<1 / n$ for every $n$. In other words, the family $(A, m)$ intersects itself on every strip of the form $0<x<1 / n$.

Since metrizability and collectionwise normality are equivalent for Moore spaces [1], the theorem follows.

3. A counterexample to (T). In [3] it was shown that statement (T) is equivalent to the statement: Every normal Moore space $X\left(\Gamma_{0}\right)$ is metrizable. (The points of $X\left(\Gamma_{0}\right)$ are the points of the punctured plane $\mathbf{R}^{2}-\{0\}$ together with the curves of $\Gamma_{0}$; the punctured plane has the discrete topology and basic neighborhoods of a point $\gamma$ in $\Gamma_{0}$ are the truncations of $\gamma$.)

ThEOREM (MA $+\neg \mathrm{CH})$. There exists a nonmetrizable normal Moore space $X\left(\Gamma_{0}\right)$.

Proof. Let $X(S, m)$ be a nonmetrizable normal picket fence space and let $\Gamma_{0}$ be the family of parabolic approaches $y=m(s) x^{2}+s x, x>0, s \in S$. Obviously, $X\left(\Gamma_{0}\right)$ is a Moore space. Normality and noncollectionwise normality follow from the fact that the truncated lines $y=m(s) x+s, 0<x<\sigma$, and $y=m(t) x+t, 0<x<\tau$, intersect if and only if the truncated parabolas $y=m(s) x^{2}+s x, 0<x<\sigma$, and $y=m(t) x^{2}+t x, 0<x<\tau$, intersect.

REMARK. In the above theorems, the full strength of $\mathrm{MA}+\neg \mathrm{CH}$ is not 
needed; all that is needed is the existence of a $Q$-set (cf., e.g., Theorem 5(b) of [7]).

\section{REFERENCES}

1. R. H. Bing, Metrization of topological spaces, Canad. J. Math. 3 (1951), 175-186. MR 13, 264.

2. J. A. Eidswick, On some fundamental problems in cluster set theory, Proc. Amer. Math. Soc. 39 (1973), 163-168. MR 47 \#2008.

3. - A topological translation of a fundamental problem in cluster set theory, Proc. Amer. Math. Soc. 53 (1975), 75-79.

4. — A crowded set of non-intersecting lines, Amer. Math. Monthly 80 (1973), 415. MR 47 \#3178.

5. D. A. Martin and R. M. Solovay, Internal Cohen extensions, Ann. Math. Logic 2 (1970), no. 2, 143-178. MR 42 \#5787.

6. I. P. Natanson, Theory of functions of a real variable, GITTL, Moscow, 1950; English transl., Vol. 1 (Chaps. 1-9), Ungar, New York,1955. MR 12, 598; 16, 804.

7. F. Rothberger, On some problems of Hausdorff and of Sierpinski, Fund. Math. 35 (1948), 29-46. MR 10, 689.

8. J. R. Shoenfield, Martin's axiom, Amer. Math. Monthly 82 (1975), 610-617.

9. L. A. Steen, Conjectures and counterexamples in metrization theory, Amer. Math. Monthly 79 (1972), 113-132. MR 46 \#8186.

Department of Mathematics, University of Nebraska, Lincoln, Nebraska 68508 